Please do not remove this page

RMIT

UNIVERSITY

\title{
Painful toxins acting at TRPV1
}

Cromer, Brett; McIntyre, Peter

https://researchrepository.rmit.edu.au/esploro/outputs/9921857960001341/filesAndLinks?institution=61RMIT_INST\&index=null

Cromer, B., \& McIntyre, P. (2008). Painful toxins acting at TRPV1. Toxicon, 51(2), 163-173.

https://doi.org/10.1016/j.toxicon.2007.10.012

Document Version: Accepted Manuscript

Published Version: https://doi.org/10.1016/j.toxicon.2007.10.012

Repository homepage: https://researchrepository.rmit.edu.au

(c) 2007 Elsevier Ltd. All rights reserved

Downloaded On 2023/04/26 20:17:00 +1000

Please do not remove this page 
Title: Painful Toxins acting at TRPV1

Authors: Brett A. Cromer ${ }^{1,2}$ and Peter McIntyre ${ }^{*}, 1$

Affiliations:

${ }^{1}$ Department of Pharmacology, University of Melbourne, Victoria 3010, Australia

${ }^{2}$ The Howard Florey Institute, University of Melbourne, Victoria 3010, Australia

\begin{abstract}
Many plant and animal toxins cause aversive behaviours in animals due to their pungent or unpleasant taste or because they cause other unpleasant senstations, like pain. This article reviews the current state of knowledge of toxins which act at the TRPV1 ion channel, which is expressed in primary sensory neurons, is activated by multiple painful stimuli and is thought to be a key pain sensor and integrator. The recent finding that painful peptide "vanillotoxin" components of tarantula toxin activate the TRPV1 ion channel to cause pain led us to survey what is known about toxins which act at this receptor. Toxins from plants, spiders and jellyfish are considered. Where possible, structural information about sites of interaction is considered in relation to toxin binding sites on the $\mathrm{Kv}$ ion channel, for which more structural information exists. We discuss a developing model where toxin agonists such as resiniferatoxin and vanillotoxins are proposed to interact with a region of TRPV1 which is homologous to the "voltage sensor" in the Kv1.2 ion channel, to open the channel and activate primary sensory nerves, causing pain.
\end{abstract}

Keywords

TRPV1, ion channel, pain, tarantula, toxin

*Corresponding author. Fax: 61-3-8344 5743 Email: pmci@unimelb.edu.au 


\section{Introduction}

Toxins may be employed defensively or aggressively. Plants are well known to discourage predation by animals through the defensive use of toxins that may have an unpleasant taste, act as irritants, cause pain or even death. Animals, conversely, are better known for their aggressive use of toxins in venoms for paralyzing and killing prey but there are also many animals, such as bees, that use toxins defensively to ward off predators or competitors, often by inflicting pain. Many painful venom components are neurotoxins that act directly on receptors or enzymes of the peripheral sensory nerves which sense pain. For example, some painful wasp venom peptide toxins are agonists of kinin receptors and the principal painful bee toxin, melittin, is a potent phospholipase A2 activator which also inhibits protein kinase C and several other protein kinases. Venomous animals that use toxins aggressively for predation have an ideal weapon that they may also use defensively, again often inflicting pain. As a mode of action for defensive toxins, inflicting pain appears ideal, but is of no apparent value in predation. Consequently it is not clear whether the painful effects of predatory venoms are simply beneficial defensive side-effects of toxins used for predation or whether these venoms include specific defensive toxins. Support for the latter alternative comes from the recent identification of peptide "vanillotoxins" (VaTxs) in tarantula venom that specifically activate the noxious heat-sensing receptor TRPV1 (Figure 1) (Siemens et al, 2006), well known as the target of capsaicin (Figure 2), the painful vanilloid toxin in "hot" chilli peppers. Thus in a surprising case of convergent evolution, chilli plants and tarantulas have evolved very different toxins that activate the same pain-sensing receptors in mammals. Further investigation will be required to determine whether there are other painful toxins that target TRPV1, or other TRP family members that also act as pain sensors. There are, however, some preliminary hints that this may be the case. The burning and tingling neurological sensations typically associated with neurotoxic shellfish poisoning and ciguatera fish poisoning are believed to be due to polyether toxins, such as gambierol and brevetoxin 
and these toxins have recently been shown to potentiate activation of TRPV1 by capsaicin (Cuypers et al 2007). Similarly, components of venom from jellyfish and other cnidaria are able to potentiate the action of capsaicin and prevent desensitization of TRPV1 (Cuypers et al, 2006) but it is not clear if this is a direct action on TRPV1 or if there would be any effect in the absence of capsaicin. The precise venom components mediating these effects are also unknown. Precise components of American funnel web venom, agatoxins (Figure 2), have been shown to block TRPV1 (Kitaguchi \& Swartz, 2005) in a manner that is proposed to be analogous to block by arginine-rich peptides (Planells-Cases et al, 2000) but this effect occurs at relatively low potency and is not specific for TRPV1. Such inhibition of TRPV1 would also cause analgesia rather than pain.

The sequence of the tarantula vanillotoxins (Figure 3) identifies them as members of the inhibitor cysteine knot (ICK) peptide family (Siemens et al, 2006), with greatest homology to the sub-family of ICK toxins that modulate voltage gating of potassium channels and are most prominent in the venom of tarantulas (Swartz, 2007). The probable close evolutionary link between this sub-family and vanillotoxins is supported by the finding that, as well as activating TRPV1, one of the vanillotoxins (VaTx1) modulates voltage gating of Kv2.1, a member of the Shab family of voltage-gated potassium (Kv) channels (Siemens et al, 2006). In this review we will present a brief summary of what is known about the biology and molecular mechanisms of TRPV1 function and the action of non-peptide plant toxins such as capsaicin. Finally we will discuss, in a more speculative manner, the possible mechanism of action of vanillotoxins on TRPV1, drawing heavily on the analogy of the effects of related ICK toxins on voltage gating of distantly related ion channels.

\section{TRPV1 biology}

TRPV1 or the capsaicin receptor, first cloned and characterized by Caterina, Julius and colleagues (Caterina et al, 1997), is a member of the transient receptor potential gene family of non-selective cation channels, which includes a number of thermosensitive ion channels 
which are involved in pain pathways, such as TRPV4 (Grant et al, 2007), TRPM8 (Bautista et al., 2007; Dahka et al 2007; Colburn et al., 2007 ) and TRPA1(Kwan et al 2006; Bautista et al., 2006). TRPV1 is expressed in pain-sensing peripheral sensory neurons and is a defining marker of these neurons, which, like TRPV1, respond to multiple painful stimuli, including noxious heat $\left(>42^{\circ} \mathrm{C}\right)$, extracellular $\mathrm{pH}$ below 5.5 and, vanilloid toxins such as capsaicin from hot chili peppers. TRPV1 from rat, human and guinea pig, is activated by heat as well as by capsaicin in the low micromolar range (McIntyre et al, 2001; Savidge et al, 2002). TRPV1 is an integrator of different painful stimuli (Tominaga et al, 1998) and has recently attracted a great deal of attention as a potential therapeutic target for treating chronic inflammatory and neuropathic pain (Culshaw et al, 2006; Gavva et al, 2005; Pomonis et al, 2003; Walker et al, 2003).

Capsaicin-sensitive peripheral sensory nerves are generally unmyelinated C-fibres or thinly myelinated $\mathrm{A} \delta$-fibres which have their cell bodies in the dorsal root ganglia and trigeminal ganglia. Nerve damage is able to induce an increase in the expression of TRPV1 in adjacent undamaged nerves and this increase is more substantial in the A $\delta$ fibres (Hudson et al, 2001) and this pattern of expression provides circumstantial evidence of the importance of TRPV1 in sensitization and pain transmission under these conditions. Other studies have demonstrated increased functional expression of TRPV1 in painful neuropathic and inflammatory conditions (Rashid et al, 2003a; Rashid et al, 2003b). Experiments with TRPV1 knockout mice have shown that TRPV1 expression is essential for development carageenaninduced inflammatory thermal hyperalgesia (Davis et al, 2000) and has deficits in capsaicinsensing and neuropathic pain signaling (Caterina et al, 2000). The competitive capsaicin antagonist capsazepine (Figure 2) was the first capsaicin antagonist that was shown to be able to inhibit chronic pain behaviors in inflammatory and neuropathic pain models (Walker et al, 2003). Notably, this effect is species-specific and is evident in guinea-pigs but not rats or mice. Subsequently others have shown that other TRPV1 antagonists, which do not show 
species selectivity are also effective in models of inflammatory and neuropathic pain (Culshaw et al, 2006; Gavva et al, 2005; Pomonis et al, 2003) as well as cancer pain (Ghilardi et al, 2005).

The regulatory lipid phosphatidylinositol-4,5-bisphosphate $\left(\mathrm{PIP}_{2}\right)$ is well known as a modulator of TRPV1 but its precise role is controversial, being described either as an inhibitor (Prescott \& Julius, 2003) or a sensitizer (Liu et al, 2005; Stein et al, 2006). The search for other endogenous activators of TRPV1 has focused on arachadonic acid metabolites and $\mathrm{N}$-acyl-dopamine derivatives, which have been found in vivo. Eicosanoid metabolites of lipoxygenase action on arachadonic acid such as 12-(S)-HPETE, 15-(S)HPETE, 5-(S)-HETE and leukotriene B4, have been proposed to be endogenous activators of TRPV1 (Hwang et al, 2000), although each of these agonists is weak ( $>8 \mu \mathrm{M})$ in in vitro assays. Other endogenous agonists of TRPV1 include anandamide (Zygmunt et al, 2000), Narachidonyl-dopamine, N-oleoyl-dopamine (De Petrocellis et al, 2004) and oleo-ethanolamide (Wang et al, 2005) and since these are more potent (in the $\mathrm{nM}$ to $\mu \mathrm{M}$ range), they may be more significant agonists. Omega-3 fatty acids have also been shown to modulate TRPV1 (Matta et al, 2007).

\section{TRPV1 protein structure and function}

The TRPV1 protein is a tetrameric (Kedei et al, 2001) integral membrane protein, which in the rat comprises 839 residues, with cytoplasmic $\mathrm{N}$ - and C-terminal tails flanking six transmembrane regions (termed S1-S6 here) and a putative pore lining region between the S5 and S6. The overall membrane topology resembles that of the extensively studied voltagedependent potassium (Kv) channels and these studies provide a basic conceptual model for understanding the membrane region of TRP channels. In particular, the membrane region can be considered in two domains, a "sensor" domain comprising S1-S4 and a channel domain comprising S5, S6 and a reentrant pore loop between S5 and S6. In Kv channels, S4 contains a number of conserved positively charged residues that sense the membrane potential and in 
concert with the rest of the sensor domain determines the strongly voltage-dependent gating. TRP channels show relatively weak voltage-dependent gating and lack most of the charges in S4 but recent studies have indicated that, at least for TRPM8, charges in S4 do act as voltage sensors in voltage-dependent gating (Voets et al, 2007). The putative role of the pore loop and S6 in lining the channel is well supported by data showing residues in these regions are determinants of ion selectivity. Two acidic residues, E600 and E648, in the pore-forming region between TM5 and TM6 are critical for the sensitivity of TRPV1 to external pH (Jordt et al, 2000) and to divalent cations (Ahern et al, 2005). The E600 residue has a significant role in $\mathrm{pH}$ modulation of sensitivity to heat and to capsaicin (Figure 1), whereas E648 is more specifically involved in the direct activation of TRPV1 by protons (Jordt et al, 2000). Channel domain structures from a number of bacterial Kv proteins have been resolved using x-ray crystallography (MacKinnon, 2003). More recently, the voltage sensor domain together with the channel domain of a mammalian Kv1.2 channel has been resolved (Long et al, 2005a). In the absence of detailed structural information for TRP channels, Kv channel structures have been used as templates for building structural homology models of TRPV1 (Brauchi et al, 2007), and we have done the same here (Figure 1B). These models are very useful for conceptual understanding of TRP channel structure and function but, given the low sequence homology with the $\mathrm{Kv}$ templates, great care must be taken in interpreting the finer details.

The TRPV1 N-terminal cytoplasmic region of approximately 430 residues is comprised primarily of six ankyrin repeats. Prior to the ankyrin repeat domain, the first $109 \mathrm{~N}$-terminal residue segment is proline-rich, likely to be unstructured and is dispensable for robust capsaicin activated currents (Jung et al, 2002). The stucture of the ankyrin repeat domain has recently been determined, including a bound ATP (Lishko et al, 2007). This bound ATP sensitizes TRPV1 to capsaicin activation (Kwak et al, 2000) and prevents desensitization with repeated activation (or tachyphylaxis) by competitively inhibiting calmodulin binding (Lishko 
et al, 2007), consistent with an overlap between the ATP-binding site and a previously identified calmodulin-binding region within the ankyrin repeat domain (Figure 1 )

(Rosenbaum et al, 2004). PIP $_{2}$ was also shown to reduce tachyphylaxis (Lishko et al, 2007), consistent with its inhibition of calmodulin binding to the TRPV1 C-terminal region (Kwon et al, 2007) and the identification of a calmodulin-binding segment in the distal C-terminus (Numazaki et al, 2003) that overlaps with a segment important for modulation by $\mathrm{PIP}_{2}$ (Prescott \& Julius, 2003). The last 72 C-terminal residues, including these two overlapping segments, are dispensable for robust capsaicin activated currents (Jung et al, 2002). Immediately following S6 in the C-terminal cytoplasmic region is a segment well conserved throughout the TRP family, known as the “TRP domain”, including a highly conserved five residue "TRP box". Two basic residues in this region have been shown to be critical for $\mathrm{PIP}_{2}$ channel sensitization in TRPM8, TRPM5, TRPV5 (Rohacs et al, 2005) and a TRPM8 chimera containing the equivalent region from TRPV1 (Brauchi et al, 2007). A region slightly larger than the TRP domain is strongly predicted by a number of secondary structure algorithms to be highly alpha-helical and is proposed to be involved in subunit interaction (Garcia-Sanz et al, 2004). Indeed, based on further secondary structure analysis, a structural model has been proposed for the complete cytoplasmic C-terminal tail of TRPV1, built using the HCN-2 cyclic nucleotide-binding domain as a template (Brauchi et al, 2007; Garcia-Sanz et al, 2004) Given the lack of sequence homology with the template, however, this model requires further experimental validation. Chimeras between the cold sensitive TRPM8 and the heat sensitive TRPV1 have demonstrated that the thermal sensitivity of these TRP channels is determined almost entirely by the C-terminal cytoplasmic region (Brauchi et al, 2007; Brauchi et al, 2006), primarily by a segment between the TRP domain and the camodulin-binding segment (Brauchi et al, 2007). Interestingly, a mutation within this segment, K735R, abolished sensitization by ATP (Kwak et al, 2000). 
In summary, the studies described above present a model of TRPV1 comprising a number of functional modules (Latorre et al, 2007), analogous to those seen in Kv channels. The channel itself, formed from the core channel module, is lined by the reentrant pore loop at the extracellular end and by S6 at the cytoplasmic end. By analogy with Kv channels, the cytoplasmic end of S6 is expected to gate the channel with an iris-like motion (Gulbis \& Doyle, 2004; Long et al, 2005b). TRPV1 and other TRP channels are only weakly voltagedependent however, like Kv channels, basic residues in S4 of the sensor module appear to act as gating charges, presumably activating the channel gate via the S4-S5 linker (Long et al, 2005b). Consistent with the Kv channel analogy (Roosild et al, 2004), cytoplasmic N- and Cterminal modules regulate gating in response to temperature and cytoplasmic ligands. Thus three different modules interact in gating the channel, with additional input from $\mathrm{pH}$ sensors on the extracellular face of the channel module.

\section{Non-peptide plant toxins that target TRPV1}

Capsaicin (Figure 2) is a pungent compound from chilli peppers, the fruit of Capsicum frutescens and is the classic vanilloid agonist of TRPV1. The early functional identification of rat TRPV1 cDNA based on activation by capsaicin (Caterina et al, 1997) is the reason for the classification of TRPV1 as the first of the vanilloid receptor subfamily of TRP channels, even though no other TRPV family members respond to vanilloid agonists. TRPV1, 2, 3 and 4 share between $37 \%$ and $47 \%$ amino acid identity and the regional similarities are significantly higher in transmembrane domains and other conserved cytoplasmic regions. Resiniferatoxin, (RTX, Figure 2) from the Euphorbia resinifera plant, is an ultra-potent vanilloid and can activate TRPV1 in the nanomolar range (Szallasi \& Blumberg, 1989). The high affinity of RTX for TRPV1 enables ${ }^{3} \mathrm{H}$-RTX to be used for receptor binding studies. Capsazepine is a synthetic analog of capsaicin (Figure 2). It was the first competitive antagonist of capsaicin identified (Walpole et al, 1994) and has been widely used to study the pharmacology of 
TRPV1. Iodo-RTX (Figure 2) a derivative of RTX is an antagonist of capsaicin and RTX with high affinity for TRPV1 (Johnson et al, 2006; Wahl et al, 2001).

Chickens are able to ingest capsaicin without displaying pain-avoiding behaviors. Indeed, chicken TRPV1 is not sensitive to capsaicin, although the channel is activated by heat and low $\mathrm{pH}$, much like mammalian TRPV1. Chimeric receptors were used to exploit this species difference, identifying S3 (see Figure 1) as a key region determining capsaicin sensitivity and more specifically, residues R491, Y511 and S512 at the base of S2 and S3 of rat TRPV1 (Jordt \& Julius, 2002). In the same study, RTX binding was found to depend on determinates within the whole segment from transmembrane two to four. Species differences between rat and human, in the ability of capsazepine to block proton activation (McIntyre et al, 2001; Walker et al, 2003), were again exploited with chimeric receptors to identify I514 and V518 in S3, and M547 in S4 as key amino acids for capsazepine interaction with TRPV1 (Phillips et al, 2004). This study also found that the human TRPV1 mutation L547M, converted rat TRPV1 agonist, phorbol 12-phenylacetate 13-acetate 20-homovanillate (PPAHV), which is inactive at human TRPV1, into an agonist as it is at the rat TRPV1 (Phillips et al, 2004). Species selectivity of capsaicin between rat and rabbit was used to map residue M547 and T550 as being responsible for the lack of responsiveness of rabbit TRPV1 to capsaicin (Gavva et al, 2004). Finally, the activation of TRPV1 by RTX is critically dependent on M547 in S4 and the S512Y mutation in S3 converts the action of iodo-RTX from an antagonist to an agonist (Johnson et al, 2006).

From these data, a picture emerges of an intra-membrane pocket between S3 and S4 in the sensor domain that has been utilized by plants as a target for toxins that cause pain by activating TRPV1, apparently by hijacking the gating function of the sensor domain. The role of this pocket in normal physiological gating of TRPV1 is not clear but it may be the target for putative endogenous agonists such as anandamide (Jordt \& Julius, 2002). 


\section{Non-peptide animal toxin interactions with TRPV1}

The venom of the American funnel web spider Agelenopsis aperta includes high concentrations of two acylpolyamine toxins, agatoxin 489 (Figure 2) and agatoxin 505, that are known to paralyse insects by inhibiting synaptic glutamate receptors (Adams, 2004; Adams et al, 1989; Skinner et al, 1989) but have recently been shown to also inhibit TRPV1. Based on the voltage-dependence of inhibition and the effect mutations in the channel lining have on toxin sensitivity, Kitaguchi and Swartz have proposed that these toxins inhibit TRPV1 by open channel block (Kitaguchi \& Swartz, 2005), consistent with the permeability of smaller polyamines through TRPV1 channels (Ahern et al, 2006). The large aromatic groups present on these polyamine toxins (Quistad et al, 1990) presumably prevent permeability, leading to channel block. Given the inhibitory effect of these two toxins on insect glutamate receptors and the effects of polyamines on a wide variety of cation channels, their effects on TRPV1 probably indicate commonality of cation channel pore structures, rather than any direct relevance of TRPV1 as a biological target for the toxins. Indeed, it is difficult to imagine what value a TRPV1 inhibitor toxin would be to a spider.

\section{Vanillotoxins; ICK peptides that activate TRPV1}

The recently identified peptide vanillotoxins (VaTxs), from tarantula venom, that activate TRPV1 show significant sequence homology to other toxins in the well-described class of inhibitor cysteine knot (ICK) peptide toxins (Siemens et al, 2006). Vanillotoxins show the closest homology to a family of the ICK toxins, including hanatoxin, that inhibit voltagegated cation channels, notably Kv channels, by modulating voltage sensitivity through interactions with the voltage sensor. Indeed, as well as activating TRPV1, VaTx1 inhibits Kv2.1 by affecting voltage gating, in a similar manner to hanatoxin, and at a similar potency to its effect on TRPV1 (Siemens et al, 2006). These data indicate that VaTx1 may have dual functions in vivo, paralyzing prey by inhibiting Kv channels and inflicting pain by activating TRPV1. VaTx2 showed a hint of this dual functionality, inhibiting Kv1.2 at high 
concentrations, whereas VaTx3 shows the greatest specificity towards TRPV1 (Siemens et al, 2006). This spectrum of specificity may provide snapshots of an evolutionary link between vanillotoxins and $\mathrm{Kv}$ voltage sensor toxins. Tarantulas are a particularly rich source of voltage sensor toxins and appear to have specialized in their production (Swartz, 2007). The three vanillotoxins identified all come from the venom of the same tarantula species, Psalmopoeus cambridgei, but the venom from another tarantula, Ornithoctonus huwena, also activates TRPV1 (Siemens et al, 2006), suggesting vanillotoxins may be more widely distributed. A toxin in the venom of $O$. huwena, huwentoxinV (Zhang et al, 2003) (TXH5 in Figure. 3), is closely related to VaTx3 and may be responsible for the activation of TRPV1. The high level of sequence homology between vanillotoxins and members of the voltage-sensor toxin family, particularly of all key cysteines involved in disulphide bonds, strongly indicates that vanillatoxins structures will be similar to those of closely related ICK toxins, enabling reliable homology models to be built (Figure. 3). Based on these apparent evolutionary links to the voltage-sensor toxins, it is certainly tempting to hypothesize that vanillotoxins activate TRPV1 by interacting with sensor domain (Siemens et al, 2006), similar to the inhibition of Kv channels by ICK voltage sensor toxins.

There are a number of pieces of evidence that support this hypothesis, if only circumstantially. Firstly, VaTx3 activation of TRPV1 shows a slight change in the slope of the voltage-conductance relationship, relative to activation by capsaicin (Siemens et al, 2006), indicating a change in the effective number of gating charges or the apparent field they move through. Capsaicin activates TRPV1 by markedly shifting the mid-point $\left(\mathrm{V}_{1 / 2}\right)$ of the voltageconductance relationship to more hyperpolarized potentials, relative to channel activity in the absence of agonist (Voets et al, 2004). As TRPV1 shows a similar $\mathrm{V}_{1 / 2}$ in the presence of either VaTx3 or capsaicin (Siemens et al, 2006), it is probable that VaTx3 also shifts the $\mathrm{V}_{1 / 2}$ to more hyperpolarized potentials. It is not precisely clear, however, whether this is the case as, unlike measurements of human TRPV1 (Voets et al, 2004), Siemens and colleagues saw 
virtually no activation of rat TRPV1 in the absence of toxin at $22-24^{\circ} \mathrm{C}$, even at $+200 \mathrm{mV}$ (Siemens et al, 2006) so a control $\mathrm{V}_{1 / 2}$ is not available. The reason for this discrepancy is not clear but it may reflect a species difference in $\mathrm{V}_{1 / 2}$ between rat and human TRPV1 or possibly be a function of expression differences between transiently (Voets et al, 2004) and stably transfected HEK293 cells (Siemens et al, 2006). Secondly, vanillotoxins are able to activate TRPV1 from the extracellular side of the membrane only (Siemens et al, 2006), which limits the potential sites of interaction to the pore loop, the extracellular ends of S5 and S6 and the extracellular face of the sensor domain. Modeling of VaTx1, by homology to the voltage-sensor toxin SGTx1, reveals a prominent hydrophobic face (Figure 3C, top) that, although not strictly conserved with voltage sensor toxins, is strongly conserved in terms of its hydrophobic nature. In voltage-sensor toxins this hydrophobic face partitions well into lipid bilayers, as demonstrated by the fluorescence of Trp31, a residue on this face that is strictly conserved (Lee \& MacKinnon, 2004; Phillips et al, 2005). Furthermore, the primary determinants of the inhibition of Kv2.1 by SGTx1 map onto this same hydrophobic face (Wang et al, 2004)(Figure. 3E). The general conservation of these features supports a related mechanism of action but there are also clear differences. Most obviously, voltage-sensor toxins inhibit $\mathrm{Kv}$ channels by stabilizing the closed state, whereas vanillotoxins activate TRPV1 by stabilizing the open state.

In conclusion the targeting of the sensor domain of TRPV1 by the plant toxins, capsaicin and resiniferatoxin, demonstrates that this is an effective mechanism for activating TRPV1. Whereas these plant toxins are strongly hydrophobic and can cross the membrane to act at a site in the inner leaflet, the spider vanillotoxins are amphiphilic and activate TRPV1 from the extracellular surface of the membrane. Future research will be required to definitively reveal whether painful toxins developed by plants and spiders have converged not only on the same target receptor but also on the same domain, albeit from the opposite sides of the cell membrane. 


\section{Acknowledgements}

BC is a Sir Randal Heymanson Fellow (Howard Florey Institute and Department of Pharmacology, University of Melbourne). Thanks to Dr Rachelle Gaudet for providing the coordinates of the Ankyrin repeat domain (2PNN) ahead of their release from the Protein Data Bank.

\section{References}

Adams, M.E., 2004. Agatoxins: ion channel specific toxins from the American funnel web spider, Agelenopsis aperta. Toxicon 43, 509-525.

Adams, M.E., Herold, E.E., Venema, V.J., 1989. Two classes of channel-specific toxins from funnel web spider venom. Journal of comparative physiology 164, 333-342.

Ahern, G.P., Brooks, I.M., Miyares, R.L., Wang, X.B., 2005. Extracellular cations sensitize and gate capsaicin receptor TRPV1 modulating pain signaling. J Neurosci 25, 5109-5116. Ahern, G.P., Wang, X., Miyares, R.L., 2006. Polyamines are potent ligands for the capsaicin receptor TRPV1. J Biol Chem 281, 8991-8995.

Brauchi, S., Orta, G., Mascayano, C., Salazar, M., Raddatz, N., Urbina, H., Rosenmann, E., Gonzalez-Nilo, F., Latorre, R., 2007. Dissection of the components for PIP2 activation and thermosensation in TRP channels. Proc Natl Acad Sci U S A 104, 10246-10251.

Brauchi, S., Orta, G., Salazar, M., Rosenmann, E., Latorre, R., 2006. A hot-sensing cold receptor: C-terminal domain determines thermosensation in transient receptor potential channels. J Neurosci 26, 4835-4840.

Cuypers, E., Yanagihara, A., Karlsson, E., Tytgat, J., 2006. Jellyfish and other cnidarian envenomations cause pain by affecting TRPV1 channels. FEBS Lett 580, 5728-5732. 
Cuypers, E., Yanagihara, A., Rainier, J.D., Tytgat, J., 2007. TRPV1 as a key determinant in ciguatera and neurotoxic shellfish poisoning

Biochem Biophys Res Commun 361, 214-217.

Garcia-Sanz, N., Fernandez-Carvajal, A., Morenilla-Palao, C., Planells-Cases, R., FajardoSanchez, E., Fernandez-Ballester, G., Ferrer-Montiel, A., 2004. Identification of a tetramerization domain in the C terminus of the vanilloid receptor. $J$ Neurosci 24, 5307-5314. Grant, A.D., Cottrell, G.S., Amadesi, S., Trevisani, M., Nicoletti, P., Materazzi, S., Altier, C., Cenac, N., Zamponi, G.W., Bautista-Cruz, F., Lopez, C.B., Joseph, E.K., Levine, J.D., Liedtke, W., Vanner, S., Vergnolle, N., Geppetti, P., Bunnett, N.W., 2007. Protease-activated receptor 2 sensitizes the transient receptor potential vanilloid 4 ion channel to cause mechanical hyperalgesia in mice. J Physiol, 578, 715-733.

Gulbis, J.M., Doyle, D.A., 2004. Potassium channel structures: do they conform? Current opinion in structural biology, 14, 440-446.

Humphrey, W., Dalke, A., Schulten, K., 1996. VMD: visual molecular dynamics. Journal of molecular graphics, 14, 33-38, 27-38.

Johnson, D.M., Garrett, E.M., Rutter, R., Bonnert, T.P., Gao Y.D., Middleton, R.E., Sutton K.G., 2006. Functional mapping of the transient receptor potential vanilloid 1 intracellular binding site. Mol Pharmacol 70, 1005-1012.

Jordt, S.E., Julius, D., 2002. Molecular basis for species-specific sensitivity to "hot" chili peppers. Cell 108, 421-430.

Jordt, S.E., Tominaga, M., Julius, D., 2000. Acid potentiation of the capsaicin receptor determined by a key extracellular site. Proc Natl Acad Sci U S A 97, 8134-8139.

Jung, J., Lee, S.Y., Hwang, S.W., Cho, H., Shin, J., Kang, Y.S., Kim, S., Oh, U., 2002. Agonist recognition sites in the cytosolic tails of vanilloid receptor 1. J Biol Chem 277, $44448-44454$. 
Kitaguchi, T., Swartz, K.J., 2005. An inhibitor of TRPV1 channels isolated from funnel Web spider venom. Biochemistry 44, 15544-15549.

Kwon, Y., Hofmann, T., Montell, C., 2007. Integration of phosphoinositide- and calmodulinmediated regulation of TRPC6. Mol Cell 25, 491-503.

Latorre, R., Brauchi, S., Orta, G., Zaelzer, C., Vargas, G., 2007. ThermoTRP channels as modular proteins with allosteric gating. Cell Calcium doi:10.1016/j.ceca.2007.04.004.

Lee, C.W., Kim, S., Roh, S.H., Endoh, H., Kodera, Y., Maeda, T., Kohno, T., Wang, J.M., Swartz, K.J., Kim, J.I., 2004. Solution structure and functional characterization of SGTx1, a modifier of Kv2.1 channel gating. Biochemistry 43, 890-897.

Lee, S.Y., MacKinnon, R., 2004. A membrane-access mechanism of ion channel inhibition by voltage sensor toxins from spider venom. Nature 430, 232-235.

Lishko, P.V., Procko, E., Jin, X., Phelps, C.B., Gaudet, R., 2007. The Ankyrin Repeats of TRPV1 Bind Multiple Ligands and Modulate Channel Sensitivity. Neuron 54, 905-918. Liu, B., Zhang, C., Qin, F., 2005. Functional recovery from desensitization of vanilloid receptor TRPV1 requires resynthesis of phosphatidylinositol 4,5-bisphosphate. J Neurosci 25, 4835-4843.

Long, S.B., Campbell, E.B., Mackinnon, R., 2005a. Crystal structure of a mammalian voltage-dependent Shaker family K+ channel. Science 309, 897-903.

Long, S.B., Campbell, E.B., Mackinnon, R., 2005b. Voltage sensor of Kv1.2: structural basis of electromechanical coupling. Science 309, 903-908.

MacKinnon, R., 2003. Potassium channels. FEBS Lett 555, 62-65.

Matta. J.A., Miyares, R.L., Ahern, G.P., 2007. TRPV1 is a novel target for omega-3 polyunsaturated fatty acids. J Physiol 578, 397-411.

McIntyre, P., McLatchie, L.M., Chambers, A., Phillips, E., Clarke, M., Savidge, J., Toms, C., Peacock, M., Shah, K., Winter, J., Weerasakera, N., Webb, M., Rang, H.P., Bevan, S., James, 
I.F., 2001. Pharmacological differences between the human and rat vanilloid receptor 1 (VR1). Br J Pharmacol 132, 1084-1094.

Numazaki, M., Tominaga, T., Takeuchi, K., Murayama, N., Toyooka, H., Tominaga, M. 2003. Structural determinant of TRPV1 desensitization interacts with calmodulin. Proc Natl Acad Sci U S A 100, 8002-8006.

Phillips, L.R., Milescu, M., Li-Smerin, Y., Mindell, J.A., Kim, J.I., Swartz, K.J., 2005. Voltage-sensor activation with a tarantula toxin as cargo. Nature 436, 857-860.

Planells-Cases, R., Aracil, A., Merino, J.M., Gallar, J., Perez-Paya, E., Belmonte, C., Gonzalez-Ros, J.M., Ferrer-Montiel, A.V., 2000. Arginine-rich peptides are blockers of VR-1 channels with analgesic activity. FEBS Lett 481, 131-136.

Prescott, E.D., Julius, D., 2003. A modular PIP2 binding site as a determinant of capsaicin receptor sensitivity. Science 300, 1284-1288.

Quistad, G.B., Suwanrumpha, S., Jarema, M.A., Shapiro, M.J., Skinner, W.S., Jamieson, G.C., Lui, A., Fu, E.W. 1990. Structures of paralytic acylpolyamines from the spider Agelenopsis aperta. Biochem Biophys Res Commun 169, 51-56.

Roberts, E., Eargle, J., Wright, D., Luthey-Schulten, Z., 2006. MultiSeq: unifying sequence and structure data for evolutionary analysis. BMC bioinformatics 7, 382.

Rohacs, T., Lopes, C.M., Michailidis, I., Logothetis, D.E., 2005. PI(4,5)P2 regulates the activation and desensitization of TRPM8 channels through the TRP domain. Nat Neurosci 8, 626-634.

Roosild, T.P., Le, K.T., Choe, S., 2004. Cytoplasmic gatekeepers of K+-channel flux: a structural perspective. Trends in biochemical sciences 29, 39-45.

Rosenbaum, T., Gordon-Shaag, A., Munari, M., Gordon, S.E., 2004. Ca ${ }^{2+} /$ calmodulin $^{2}$ modulates TRPV1 activation by capsaicin. J Gen Physiol 123, 53-62. 
Siemens, J., Zhou, S., Piskorowski, R., Nikai, T., Lumpkin, E.A., Basbaum, A.I., King, D., Julius, D., 2006. Spider toxins activate the capsaicin receptor to produce inflammatory pain. Nature 444, 208-212.

Skinner, W.S., Adams, M.E., Quistad, G.B., Kataoka, H., Cesarin, B.J., Enderlin, F.E., Schooley, D.A., 1989. Purification and characterization of two classes of neurotoxins from the funnel web spider. Agelenopsis aperta. J Biol Chem 264, 2150-2155.

Stein, A.T., Ufret-Vincenty, C.A., Hua, L., Santana, L.F., Gordon, S.E., 2006.

Phosphoinositide 3-kinase binds to TRPV1 and mediates NGF-stimulated TRPV1 trafficking to the plasma membrane. J Gen Physiol 128, 509-522.

Swartz, K.J., 2007. Tarantula toxins interacting with voltage sensors in potassium channels. Toxicon 49, 213-230.

Szallasi, A., Blumberg, P.M., 1989. Resiniferatoxin, a phorbol-related diterpene, acts as an ultrapotent analog of capsaicin, the irritant constituent in red pepper. Neuroscience 30, 515520.

Voets, T., Droogmans, G., Wissenbach, U., Janssens, A., Flockerzi, V., Nilius, B., 2004. The principle of temperature-dependent gating in cold- and heat-sensitive TRP channels. Nature 430, 748-754.

Voets, T., Owsianik, G., Janssens, A., Talavera, K., Nilius, B., 2007. TRPM8 voltage sensor mutants reveal a mechanism for integrating thermal and chemical stimuli. Nat Chem Biol 3, 174-182.

Walker, K.M., Urban, L., Medhurst, S.J., Patel, S., Panesar, M., Fox, A.J., McIntyre, P., 2003. The VR1 antagonist capsazepine reverses mechanical hyperalgesia in models of inflammatory and neuropathic pain. J Pharmacol Exp Ther 304, 56-62.

Wang, J.M., Roh, S.H., Kim, S., Lee, C.W., Kim, J.I., Swartz, K.J., 2004. Molecular surface of tarantula toxins interacting with voltage sensors in K(v) channels. J Gen Physiol 123, 455467. 
Wang, X., Miyares, R.L., Ahern, G.P., 2005. Oleoylethanolamide excites vagal sensory neurones, induces visceral pain and reduces short-term food intake in mice via capsaicin receptor TRPV1. J Physiol 564, 541-547.

Zhang, P.F., Chen, P., Hu, W.J., Liang, S.P., 2003. Huwentoxin-V, a novel insecticidal peptide toxin from the spider Selenocosmia huwena, and a natural mutant of the toxin: indicates the key amino acid residues related to the biological activity. Toxicon 42, 15-20. Zygmunt, P.M., Julius, I., Di Marzo, I., Hogestatt, E.D., 2000. Anandamide - the other side of the coin. Trends Pharmacol Sci 21, 43-44. 


\section{Figure Legends:}

Figure 1. Schematic structural model of TRPV1. Grey lines delineate the cell membrane, with the extracellular side at the top and the cytoplasmic side at the bottom. A. Simple topological model of TRPV1 with cytoplasmic N- and C-termini, including ankyrin repeats (lime green) in the N-terminal region, and an integral membrane region that can be split into two parts, the sensor domain comprising S1 (dark blue), S2 (Cyan), S3 (green) and S4 (yellow) and the channel domain comprising S5 (orange), reentrant pore loop including a pore helix (red) and S6 (magenta). Labels show the approximate location of some key residues determining capsaicin sensitivity. B. Backbone ribbon structural model of TRPV1 showing one subunit in colour (as for topology diagram in panel A) and in grey, the membrane region of a second opposite subunit from a tetramer. The other two subunits in the tetramer are not shown for clarity. The N-terminal ankyrin-repeat domain (lime green) is from the recently resolved structure, 2PNN (Lishko et al, 2007), including bound ATP (shown as sticks colored by atom). The integral membrane domains (colored as in A), built by homology with the Kv1.2 structure, 2A79 (Long et al, 2005a), are the "sensor” domain comprising S1-S4 and the channel domain comprising S5, pore loop and S6. Calmodulin-binding segments identified within the $\mathrm{N}$ - and C-terminal regions are show in chocolate brown. Immediately following S6 is the helical segment involved in subunit interactions (hot pink) that includes the TRP domain (black) and the TRP box (white). This is followed by the temperature sensing region (blue stripe within red) and the $\mathrm{PIP}_{2}$-sensitive segment (yellow) within the C-terminal calmodulin-binding segment (brown).

Figure 2. Small agonists and antagonists of TRPV1. Agonists: Capsaicin (N-[(4-hydroxy3-methoxy-phenyl) methyl]-8-methyl-non-6-enamide); Resiniferatoxin; Antagonists: 
Capsazepine (N-[2-(4-chlorophenyl)ethyl]-9,10-dihydroxy-5-azabicyclo[5.4.0]undeca-7,9,11triene-5-carbothioamide); Agatoxin 489; Iodo-resiniferatoxin

Figure 3. Structural model of vanillotoxins (based on SGTx1). A. Alignment of vanillotoxins with a set of related ICK toxins that modulate voltage gating of cation channels, including three for which the atomic structure has been resolved. The alignment is colored according to the sequence entropy (strict) scale of MultiSeq within VMD software (Humphrey et al, 1996; Roberts et al, 2006), with most conserved residues blue and least conserved red. Numbering at top is for VaTx1. B. Evolutionary tree based on sequence similarity from panel A, showing vanillotoxins (green), structurally resolved toxins (blue) and others (red). C, D \& E. Surface representation of a structural model of VaTx1 built by homology to the structure of SGTx1 (1LA4), (Lee et al, 2004), showing two views rotated by $180^{\circ}$ around the y-axis relative to one another(Lee et al, 2004), with key residues on the hydrophobic face labeled in panel D. Coloring in $\mathbf{C}$ is by side-chain type, with basic (blue), acidic (red), polar (yellow), non-polar (dark green) and tyrosine (light green); in D by degree of conservation as shown in panel A, with most conserved (blue) and least conserved (red); in E highlights (magenta) residues equivalent to those in SGTx1 that when mutated, cause a more than ten-fold change in toxin potency (Wang et al, 2004). All panels of this figure were created using MultiSeq and VMD (Humphrey et al, 1996; Roberts et al, 2006). 
A

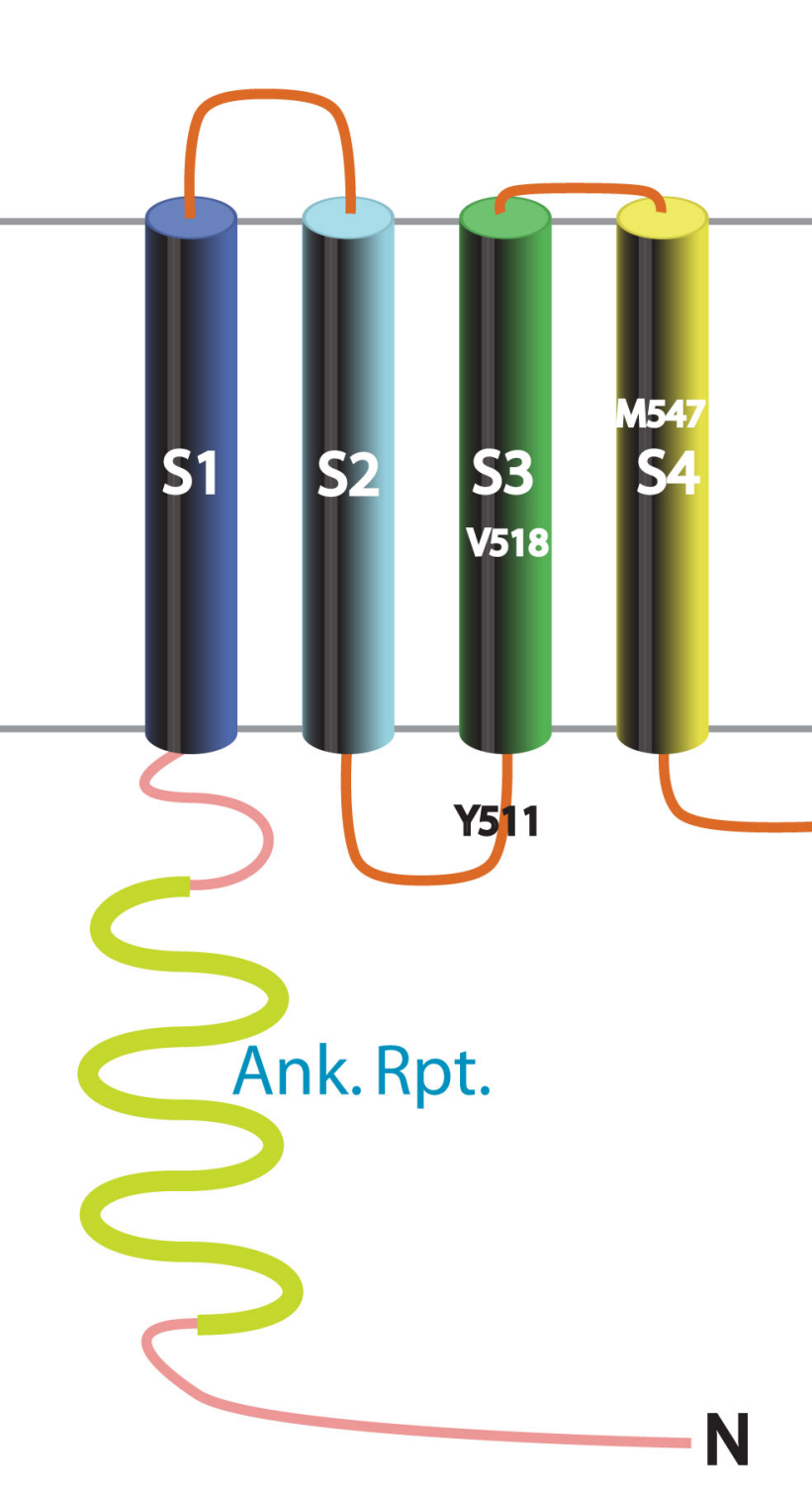

B

Extracellular

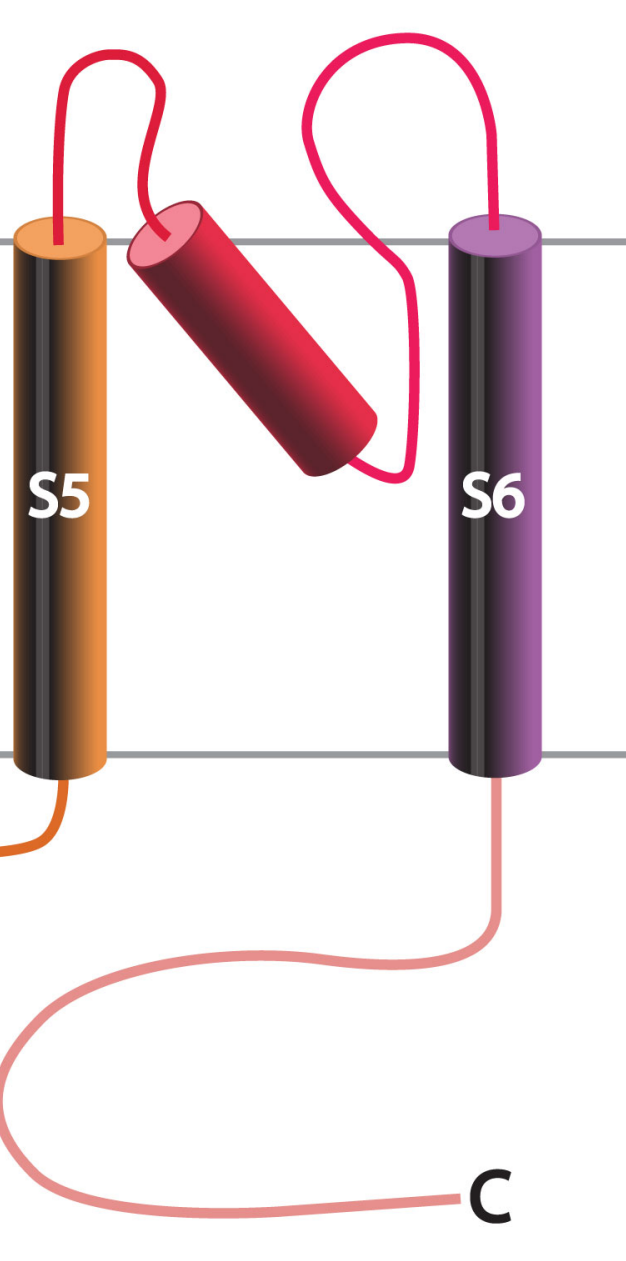

\section{M}

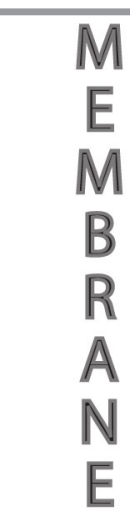

Cytoplasm

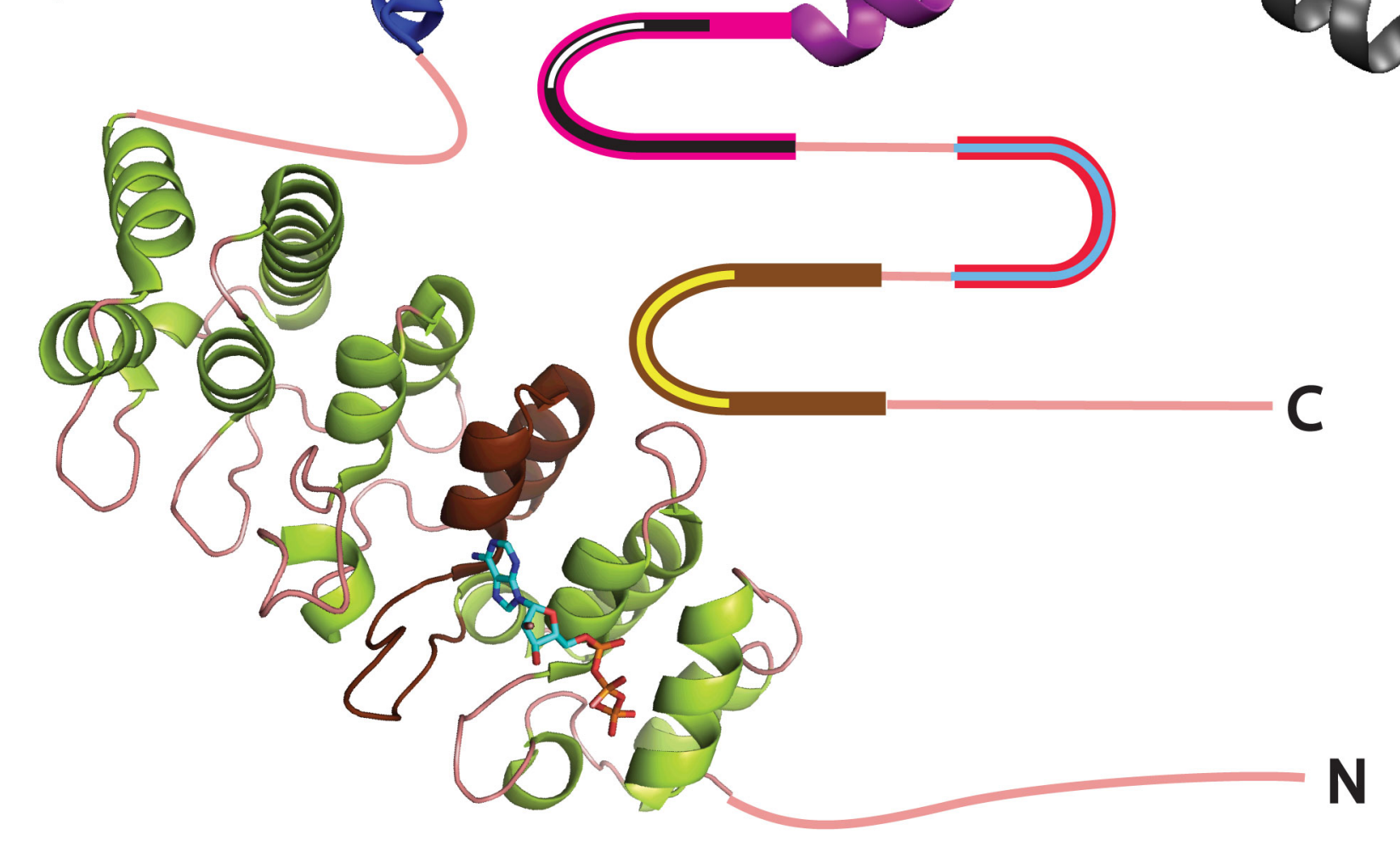



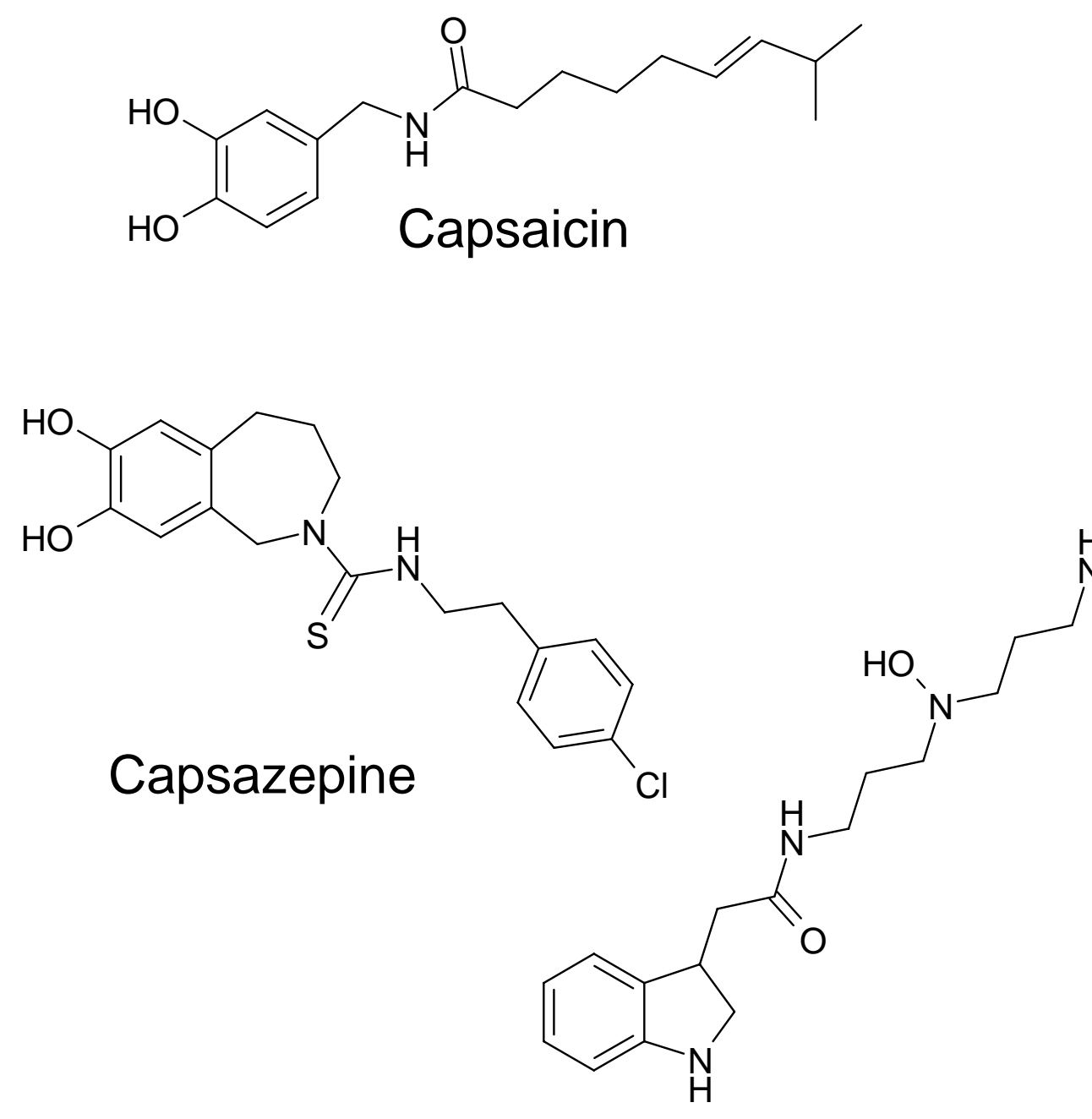

Agatoxin 489

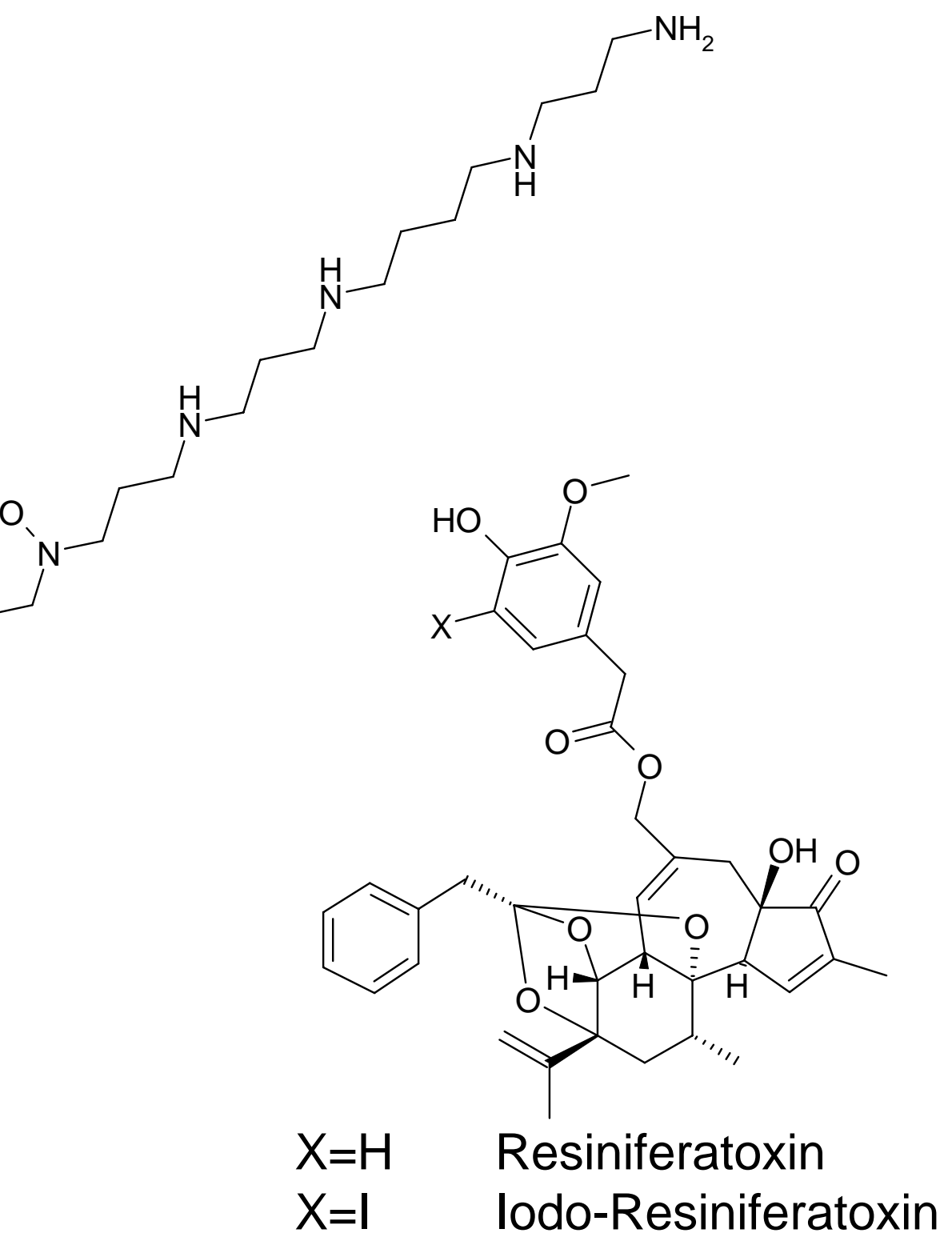


A

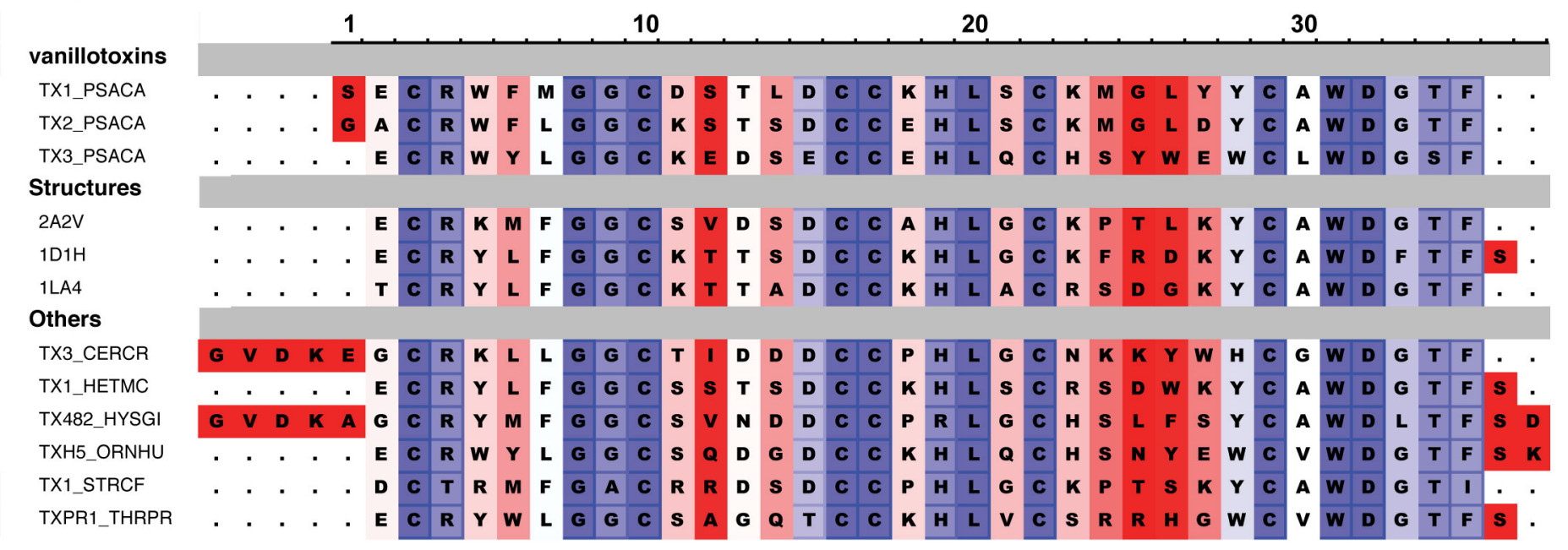

C
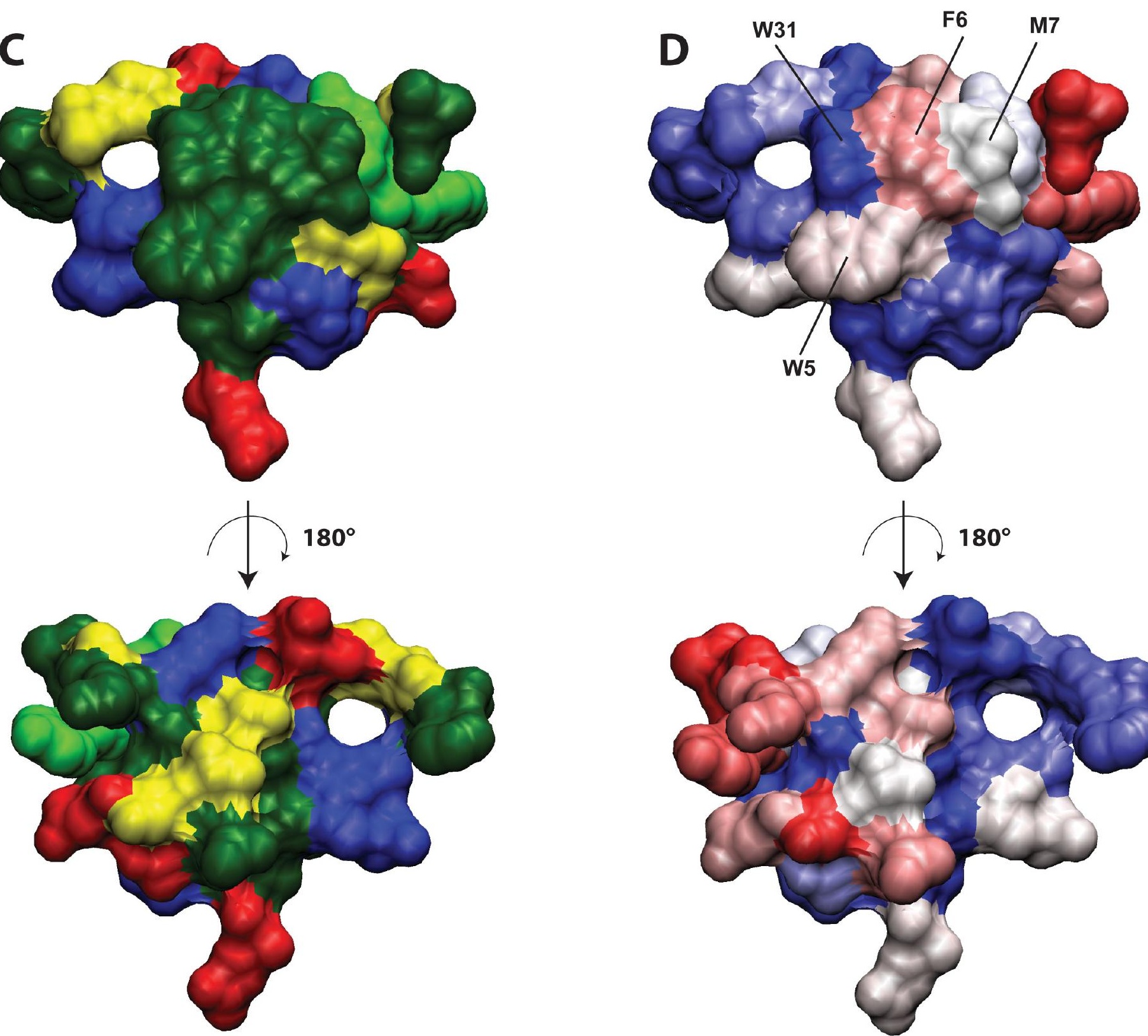

B
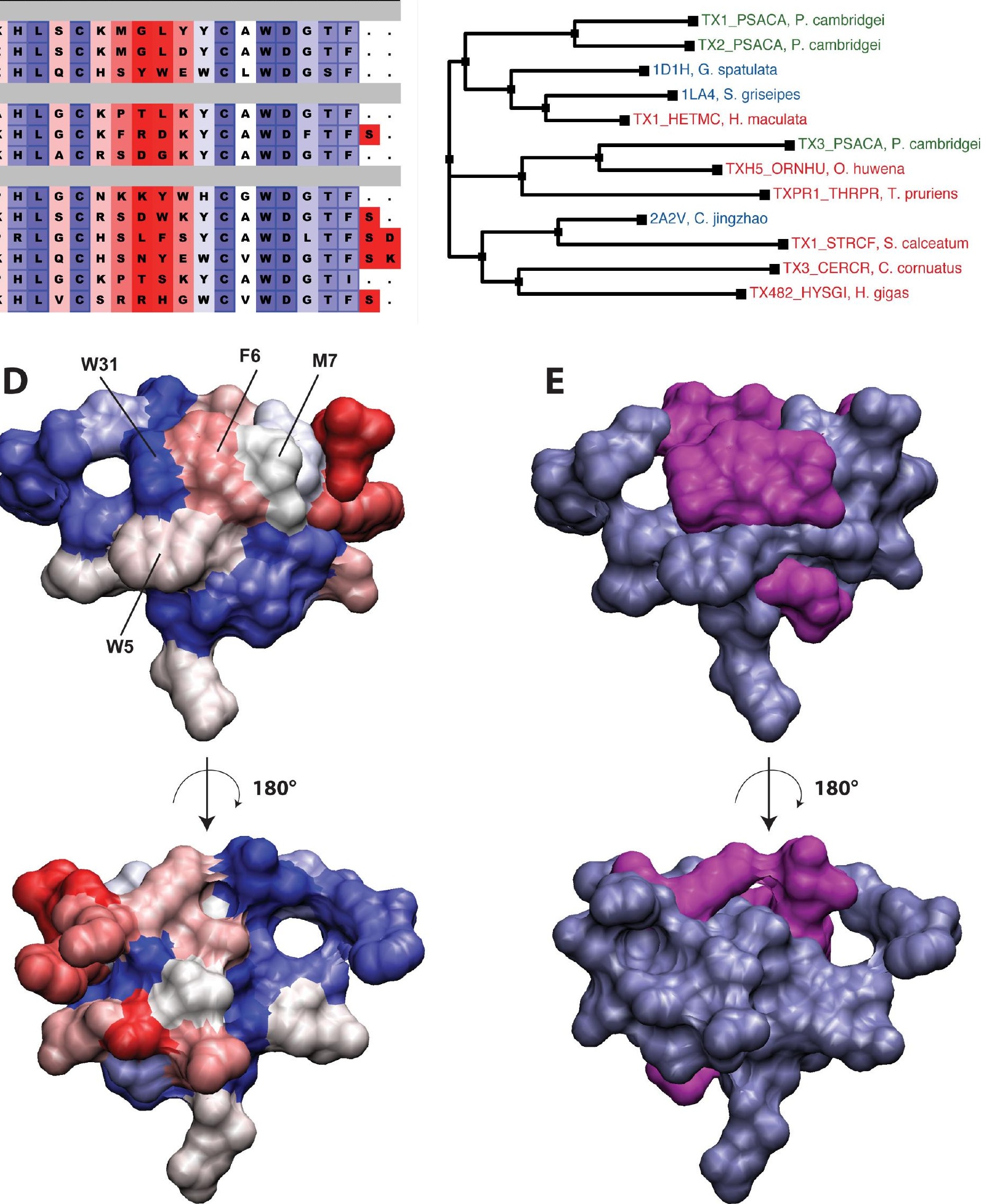